\title{
Study on Evolution Characteristics and Instability Model of Overburden Stress Shell with the Longwall Mining along Strike
}

\author{
Wanrong Liu ${ }^{1,2}$ and Qingwen Shi iD $^{3}$ \\ ${ }^{1}$ School of Architecture \& Civil Engineering, Liaocheng University, Liaocheng, Shandong 252059, China \\ ${ }^{2}$ State Key Laboratory of Mining Response and Disaster Prevention and Control in Deep Coal Mine, \\ Anhui University of Science and Technology, Huainan, Anhui 232001, China \\ ${ }^{3}$ Department of Coal Mining \& Designing, Tiandi Science \& Technology Co., Ltd., Beijing 100013, China
}

Correspondence should be addressed to Qingwen Shi; wvuqingwenshi@gmail.com

Received 26 June 2021; Accepted 20 July 2021; Published 2 August 2021

Academic Editor: Xuepeng Zhang

Copyright (c) 2021 Wanrong Liu and Qingwen Shi. This is an open access article distributed under the Creative Commons Attribution License, which permits unrestricted use, distribution, and reproduction in any medium, provided the original work is properly cited.

\begin{abstract}
Roof fracture is one of the main reasons for dynamic disaster in working face. During the process of long wall mining, due to the continuous fracture and collapse of roof, a stress shell composed of high stress is formed around the surrounding rock of the stope. The gravity of the overlying rock is transferred to the coal body around the stope through the stress shell. With the advancing of the working face, the stress shell has undergone the dynamic evolution process of the initial shell, extension shell, and flat shell. Based on the theory of shell and plate, the mechanical model of elliptical thin rock plate is constructed. The stress and bending moment distribution expressions of elliptic structure are obtained under two boundary conditions: the surrounding fixed-supported edges and simply supported edges. The results show that cracks begin to appear at the middle point of the semimajor axis and propagate along both ends of the semimajor axis. Then the fracture begins at the end of the semiminor axis, and the crack extends along the arc direction, forming an "X-O" type fracture. Finally, the mechanical nature of the stress shell instability is the result of the tensile fracture of the bedrock at the top of the shell.
\end{abstract}

\section{Introduction}

The fracture of the roof in coal mining is the main source of pressure in working face, and it is also one of the causes of dynamic disaster. For a long time, the analysis of stope pressure has been one of the hot issues of coal workers. The movement of overlying strata is closely related to the mine pressure. The movement form of overlying strata determines the appearance degree of mine pressure, and the appearance of mine pressure is also the concrete manifestation of the surrounding rock activity $[1,2]$. Scholars have done a lot of research on the mechanical characteristics of surrounding rock of coal seam mining and got a lot of meaningful conclusions. [3-5]. Shi et al. [6] studied the evolution law of overburden stress and fracture in horizontal coal seam mining. Chen et al. [7] used numerical simulation to study the three-dimensional distribution characteristics of overlying rock fractures in longwall mining. Kumar et al. [8] studied the mechanical characteristics of surrounding rock in deep thick coal seam mining. Wang et al. [9] analyzed the evolution characteristics of overburden composite pressure arch in shallow coal mining. Zhu et al. [10] studied the evolution characteristics of floor stress distribution in coal seam mining. The theories explain some mine pressure phenomena, but they do not consider the three-dimensional spatial structure of stope, which has a certain deviation from the reality. In view of the typical layered stratum structure, the small deflection thin plate theory is applied to obtain the stope roof breaking mode and evolution law [11-16]. However, the influence of the high stress concentration area formed in the three-dimensional overburden space of the stope on the failure of roof strata is not considered. Moreover, with the gradual breaking of the overlying strata, the arc structure is formed due to the breaking of the right 
angle of the roof strata, and the whole strata above the goaf form an elliptical structure. Xie et al. [17-19] found that there was a stress shell composed of high stress in the surrounding rock of the stope by using the comprehensive research methods of numerical simulation, similar simulation, and field measurement. The research shows that the stress shell is the main bearing capacity system of the overlying rock mass of the working face, which bears and transmits the stope pressure. The formation mechanism and evolution characteristics of the stress shell are related to the working face length, mining height, lithology, and other parameters, but the influence of the overlying rock fracture on the stress shell is ignored in the analysis. Therefore, based on previous studies, the authors analyze the evolution process of the stress shell and establish the fracture mechanical model of the top strata of the stress shell. The mechanical criterion of rock fracture instability is analyzed and deduced, and the fracture mode and evolution law of rock at the top of shell are revealed. Finally, the instability mechanism of stress shell is obtained.

\section{Mechanical Model of Stress Evolution of Surrounding Rock in Stope}

The comprehensive research shows that the stress shell has the following characteristics $[17,18]$. Due to the continuous mining of the coal seam, the stress balance of the original rock is destroyed under the mining disturbance, the stress of the surrounding rock is redistributed, and the mining high stress forms a stress shell in the surrounding rock space. The stress shell moves and evolves with the advance of the working face. The shell foundation of the stress shell acts on the peak area of abutment pressure in the front, rear, and flank of the working face. The stress of the shell foundation is the abutment pressure around the stope. Moreover, the stress shell exists in the overburden which has not been fractured (i.e., in the bending subsidence zone), and its height is greater than the maximum height of mining fracture development. The stress shell starts to form the initial stress shell (as shown in Figure 1, OA) from a certain distance of mining. With the continuous advance of the working face, the direct roof collapses successively, and the basic roof is broken and sunk. The stress shell evolves and develops above the rock layer and in front of the coal seam wall, forming the expansion shell (as shown in Figure 1, OB). The top of stress shell is located in the bending subsidence zone and above the fracture zone. With the continuous advance of working face, the expanded shell is easy to evolve into flat shell (as shown in Figure 1, OD). With the increase of the span of the flat shell, when the ultimate span of the rock layer at the top of the shell is reached, the roof breaks, which directly causes the stress shell to be damaged at the top of the shell. Finally, the stress shell is unstable. Because there is no stress shell to bear the gravity of the overlying rock, the stress shell instability and the whole overburden load directly will cause severe mine pressure. During the whole mining process, the stress shell experienced the dynamic evolution process of

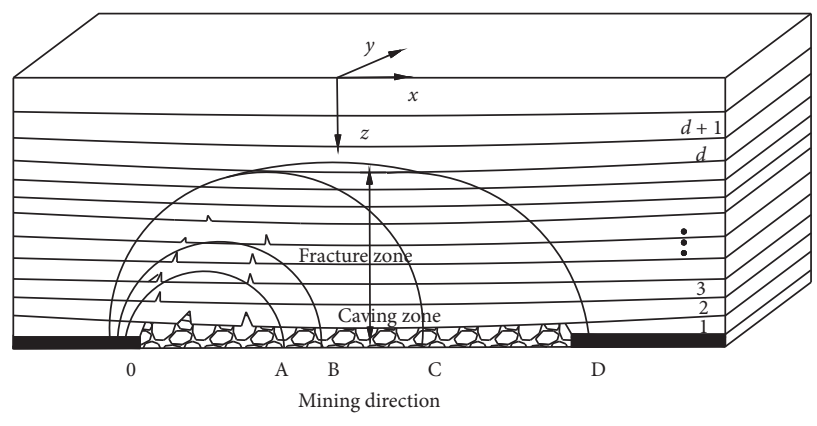

FIgURE 1: Stress shell evolution model.

the initial shell-expansion shell-flat shell along with the advancing of the working face.

When the rock stratum where the stress shell is located breaks, it will inevitably lead to the instability of the stress shell. Therefore, it is of great significance to study the fracture law of the strata at the top of the shell for mastering the instability mode of the stress shell. The intersecting line of stress shell and strata is ellipse, and the ellipse thin rock plate structure is formed in the strike and dip direction of $3 \mathrm{D}$ stope. The fracture mechanics model of shell roof strata is constructed, as shown in Figure 2.

The instability of stress shell is mainly related to the fracture of basic roof rock, so it is necessary to carry out the mechanical analysis of elliptical thin plate of shell roof rock. In the overlying strata of near horizontal coal seam (the influence of coal seam dip angle on the model is ignored in calculation), the calculation of elliptical thin rock plate size is shown in Figure 3.

It can be seen from Figure 3 that the strike length of the elliptical thin plate is as follows:

$$
m=L-2 h_{0} \cot \theta_{1},
$$

where $L$ is the advancing distance of working face; $h_{0}$ is the sum of the heights of the fault zone and the caving zone; $\theta_{1}$ is the fault angle along strike direction.

The dip length of the elliptical thin plate is as follows:

$$
n=L_{0}-h_{0}\left(\cot \theta_{2}+\cot \theta_{3}\right),
$$

where $L_{0}$ is the length of working face. $\theta_{2}$ and $\theta_{3}$ are the fracture angles below and above the coal seam along the dip direction.

\section{Mechanical Analysis of Instability Criterion}

\subsection{Failure Analysis of Fixed Support of the Rock Stratum.} Because the ellipse structure formed by the roof rock is fixed around before failure, it can be regarded as the ellipse structure fixed. The rectangular coordinate system is established. The origin is in the center of the elliptical structure, the $x$-axis is the direction of coal seam direction, and the $y$-axis is inclined direction. The elliptical structure is subjected to the combined action of gravity and stress of overlying rock. The mechanical model of elliptical structure with four sides fixed is established, as shown in Figure 4 The semimajor axis of the ellipse is 


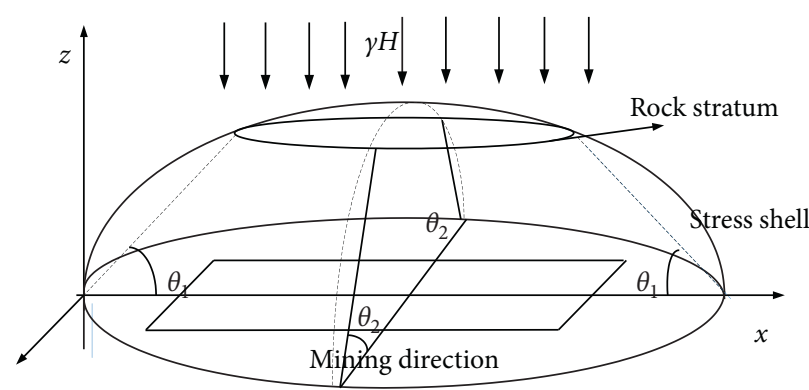

Figure 2: Fracture mechanics model of shell roof strata.

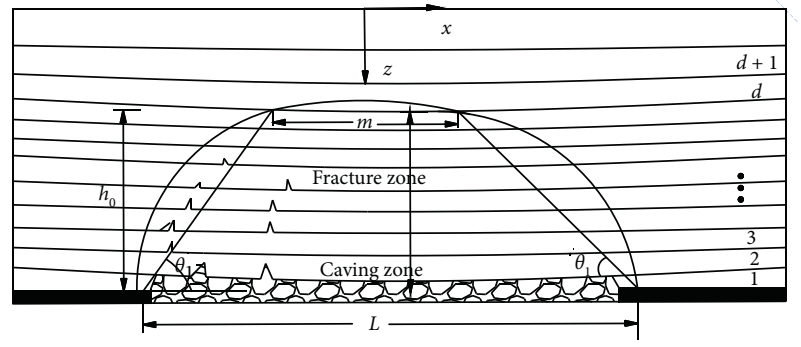

(a)

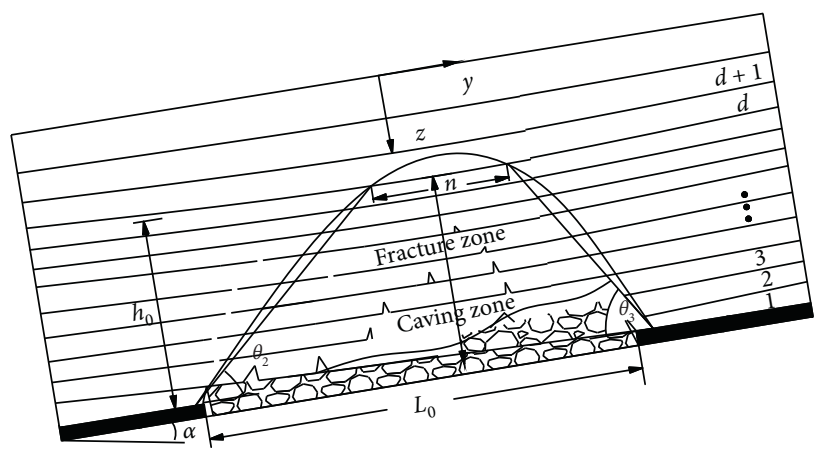

(b)

FIGURE 3: Schematic diagram for calculation of size of elliptical thin plates along strike and dip. (a) strike; (b) dip.

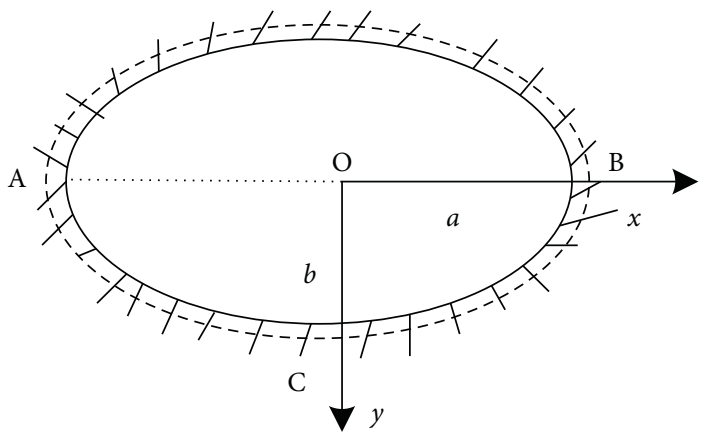

FIgURE 4: The mechanical model of the fixed-supported elliptical structure.

$a: a=m / 2$; and the semiminor axis is $b: b=n / 2$ (where $b<a)$

The boundary condition of fixed support is that the rotation angle is 0 and the displacement is 0 . Therefore, the boundary conditions of elliptic structure are as follows:

$$
\begin{aligned}
& \left.\omega_{1}\right|_{\left(x^{2} / a^{2}\right)+\left(y^{2} / b^{2}\right)=1}=0, \\
& \left.\frac{\partial \omega_{1}}{\partial x}\right|_{\left(x^{2} / a^{2}\right)+\left(y^{2} / b^{2}\right)=1}=0, \\
& \left.\frac{\partial \omega_{1}}{\partial y}\right|_{\left(x^{2} / a^{2}\right)+\left(y^{2} / b^{2}\right)=1}=0 .
\end{aligned}
$$

Take the deflection equation satisfying all boundary conditions as follows:

$$
\omega_{1}=A_{1}\left(\frac{x^{2}}{a^{2}}+\frac{y^{2}}{b^{2}}-1\right)^{2}
$$

where $A_{1}$ is a constant.

The bending equation of thin plate is as follows:

$$
\begin{aligned}
\nabla^{4} \omega_{1} & =\frac{q}{D}, \\
D & =\frac{E h^{3}}{12\left(1-\mu^{2}\right)},
\end{aligned}
$$

where $\nabla$ is Laplacian, $q$ is the gravity of overlying strata, $D$ is the bending stiffness, $h$ is the thickness of rock stratum, $\mu$ is Poisson's ratio, $E$ is the elastic modulus, and $\omega_{1}$ is the deflection of the rock stratum. 
The constant $A_{1}$ can be obtained from formula (5).

$$
A_{1}=\frac{q}{8 D\left(3 / a^{4}+2 / a^{2} b^{2}+3 / b^{4}\right)} \text {. }
$$

The deflection equation of the elliptic thin plate is obtained as follows:

$$
\omega_{1}=\frac{q\left(\left(x^{2} / a^{2}+y^{2} / b^{2}\right)-1\right)^{2}}{8 D\left(3 / a^{4}+2 / a^{2} b^{2}+3 / b^{4}\right)} .
$$

The maximum deflection occurs at the center of the elliptical rock stratum and its value is as follows:

$$
\left.\omega_{1}\right|_{x=y=0}=\frac{q}{8 D\left(3 / a^{4}+2 / a^{2} b^{2}+3 / b^{4}\right)} .
$$

Taking equation (7) into the expression of bending moment of elliptical rock stratum, the bending moment of each point of elliptical rock stratum is obtained as follows:

$$
\begin{aligned}
& M_{x}=-D\left(\frac{\partial^{2} \omega}{\partial x^{2}}+\mu \frac{\partial^{2} \omega}{\partial y^{2}}\right)=-D\left[\left(\frac{4 A_{1}}{a^{2}}+\frac{4 \mu A_{1}}{b^{2}}\right)\left(\frac{x^{2}}{a^{2}}+\frac{y^{2}}{b^{2}}-1\right)+\left(\frac{8 A_{1} x^{2}}{a^{4}}+\frac{8 \mu A_{1} y^{2}}{b^{4}}\right)\right] \\
& M_{y}=-D\left(\frac{\partial^{2} \omega}{\partial y^{2}}+\mu \frac{\partial^{2} \omega}{\partial x^{2}}\right)=-D\left[\left(\frac{4 \mu A_{1}}{a^{2}}+\frac{4 A_{1}}{b^{2}}\right)\left(\frac{x^{2}}{a^{2}}+\frac{y^{2}}{b^{2}}-1\right)+\left(\frac{8 \mu A_{1} x^{2}}{a^{4}}+\frac{8 A_{1} y^{2}}{b^{4}}\right)\right] .
\end{aligned}
$$

Then, the internal force of each point of elliptical rock stratum is obtained.

$$
\begin{aligned}
& \sigma_{x}=-\frac{E z}{1-\mu^{2}}\left(\frac{\partial^{2} \omega}{\partial x^{2}}+\mu \frac{\partial^{2} \omega}{\partial y^{2}}\right)=-\frac{E z}{1-\mu^{2}} \cdot\left[\left(\frac{4 A_{1}}{a^{2}}+\frac{4 \mu A_{1}}{b^{2}}\right)\left(\frac{x^{2}}{a^{2}}+\frac{y^{2}}{b^{2}}-1\right)+\left(\frac{8 A_{1} x^{2}}{a^{4}}+\frac{8 \mu A_{1} y^{2}}{b^{4}}\right)\right], \\
& \sigma_{y}=-\frac{E z}{1-\mu^{2}}\left(\frac{\partial^{2} \omega}{\partial y^{2}}+\mu \frac{\partial^{2} \omega}{\partial x^{2}}\right)=-\frac{E z}{1-\mu^{2}} \cdot\left[\left(\frac{4 \mu A_{1}}{a^{2}}+\frac{4 A_{1}}{b^{2}}\right)\left(\frac{x^{2}}{a^{2}}+\frac{y^{2}}{b^{2}}-1\right)+\left(\frac{8 \mu A_{1} x^{2}}{a^{4}}+\frac{8 A_{1} y^{2}}{b^{4}}\right)\right], \\
& \tau_{x y}=-\frac{E z}{1+\mu} \frac{\partial^{2} \omega}{\partial x \partial y}=-\frac{E z}{1+\mu} \frac{8 A_{1} x y}{a^{2} b^{2}} .
\end{aligned}
$$

Based on the bending moment expression (9) and stress expression (10), the maximum moment and stress are obtained at the center of the elliptical rock stratum. According to the strength characteristics of the rock, $\sigma_{\text {tensile }}<$ $\sigma_{\text {shear }}<\sigma_{\text {compressive, }}$ the elliptical rock stratum breaks, and cracks appear at the place where the tensile stress is the largest, and the cracks trace extends along the $x$-axis.

3.2. Failure Analysis of Simple Support of the Rock Stratum. According to the previous analysis, when the rock stratum is broken along the $x$-axis direction, the simply supported edge is formed along the $x$-axis direction, as shown in AB edge in Figure 5. The new model is shown in Figure 5 (due to both sides being symmetrical, one side is taken for analysis), in which the arc ACB is the fixed-support side.

The boundary conditions of simple support of the rock stratum are as follows:

$$
\begin{aligned}
\left.\omega_{2}\right|_{\left(x^{2} / a^{2}\right)+\left(y^{2} / b^{2}\right)=1(y>0)} & =0, \\
\left.\omega_{2}\right|_{y=0} & =0, \\
\left.\frac{\partial^{2} \omega_{1}}{\partial y^{2}}\right|_{y=0} & =0,
\end{aligned}
$$

$$
\left.\frac{\partial \omega_{1}}{\partial \mathrm{y}}\right|_{\left(x^{2} / a^{2}\right)+\left(y^{2} / b^{2}\right)=1(y>0)}=0 .
$$

The deflection equation of a semielliptical rock stratum satisfying all boundary conditions is as follows:

$$
\omega_{2}=A_{2} y\left(\left(\frac{x^{2}}{a^{2}}+\frac{y^{2}}{b^{2}}\right)-1\right)^{2},
$$

where $A_{2}$ is a constant.

Based on the basic assumption of thin plate bending, the total potential energy of rock stratum bending is obtained by 


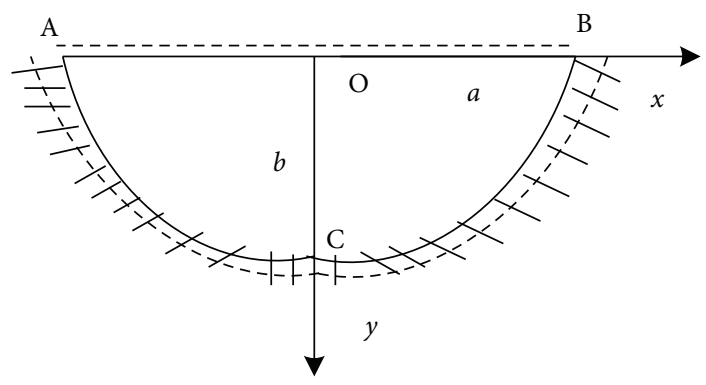

FIgure 5: The mechanical model of simple support of the rock stratum.

Rayleigh-Ritz method without considering the strain component [20].

$$
E_{P}=\frac{D}{2} \iint\left\{\left(\frac{\partial^{2} \omega}{\partial x^{2}}+\frac{\partial^{2} \omega}{\partial y^{2}}\right)^{2}-2(1-\mu)-\left[\frac{\partial^{2} \omega}{\partial x^{2}} \frac{\partial^{2} \omega}{\partial y^{2}}-\left(\frac{\partial^{2} \omega}{\partial x \partial x}\right)^{2}\right]\right\} \mathrm{d} x \mathrm{~d} y-\iint q \omega \mathrm{d} x \mathrm{~d} y
$$

Take the deflection in equation (13) into equation (12) and get the following formula:

$$
E_{P}=\frac{D}{2} A_{2}^{2} \frac{32 \cdot\left(123 a^{4}+122 a^{2} b^{2}+43 b^{4}\right)}{105 a^{3} b}-A_{2} q \frac{a b^{2}}{5} .
$$

The following formula is obtained according to the principle of minimum potential energy:

$$
\frac{\partial E_{P}}{\partial A_{2}}=0 .
$$

The $A_{2}$ value is obtained from formulas (14) and (15) as

$$
A_{2}=\frac{21 q}{32 D} \frac{a^{4} b^{3}}{\left(123 a^{4}+122 a^{2} b^{2}+43 b^{4}\right)} .
$$

So the deflection equation is as follows:

$$
\omega_{2}=\frac{21 q}{32 D} \frac{a^{4} b^{3}}{\left(123 a^{4}+122 a^{2} b^{2}+43 b^{4}\right)} y\left(\left(\frac{x^{2}}{a^{2}}+\frac{y^{2}}{b^{2}}\right)-1\right)^{2} \text {. }
$$

According to the internal force and bending moment formula of elasticity, the bending moment formula of equal thickness rock stratum is obtained as follows: follows:

$$
\begin{aligned}
& M_{x}=-D\left(\frac{\partial^{2} \omega}{\partial x^{2}}+\mu \frac{\partial^{2} \omega}{\partial y^{2}}\right)=-D\left[\left(\frac{12 A_{2} \mu y}{b^{2}}+\frac{4 A_{2} y}{a^{2}}\right)\left(\left(\frac{x^{2}}{a^{2}}+\frac{y^{2}}{b^{2}}\right)-1\right)+\left(\frac{8 A_{2} \mu y^{3}}{b^{4}}+\frac{8 A_{2} x^{2} y}{a^{4}}\right)\right], \\
& M_{y}=-D\left(\frac{\partial^{2} \omega}{\partial y^{2}}+\mu \frac{\partial^{2} \omega}{\partial x^{2}}\right)=-D\left[\left(\frac{12 A_{2} y}{b^{2}}+\frac{4 A_{2} \mu y}{a^{2}}\right)\left(\left(\frac{x^{2}}{a^{2}}+\frac{y^{2}}{b^{2}}\right)-1\right)+\left(\frac{8 A_{2} y^{3}}{b^{4}}+\frac{8 A_{2} \mu x^{2} y}{a^{4}}\right)\right] .
\end{aligned}
$$

According to the stress derivation formula of elasticity, the expression of stress component of equal thickness rock stratum is obtained as follows:

$$
\begin{aligned}
\sigma_{x} & =-\frac{E z}{1-\mu^{2}}\left(\frac{\partial^{2} \omega}{\partial x^{2}}+\mu \frac{\partial^{2} \omega}{\partial y^{2}}\right) \\
& =-\frac{E z}{1-\mu^{2}}\left[\left(\frac{12 A_{2} \mu y}{b^{2}}+\frac{4 A_{2} y}{a^{2}}\right)\left(\frac{x^{2}}{a^{2}}+\frac{y^{2}}{b^{2}}-1\right)+\frac{8 A_{2} \mu y^{3}}{b^{4}}+\frac{8 A_{2} x^{2} y}{a^{4}}\right]
\end{aligned}
$$




$$
\begin{aligned}
\sigma_{y} & =-\frac{E z}{1-\mu^{2}}\left(\frac{\partial^{2} \omega}{\partial y^{2}}+\mu \frac{\partial^{2} \omega}{\partial x^{2}}\right) \\
& =-\frac{E z}{1-\mu^{2}}\left[\left(\frac{12 A_{2} y}{b^{2}}+\frac{4 A_{2} \mu y}{a^{2}}\right)\left(\frac{x^{2}}{a^{2}}+\frac{y^{2}}{b^{2}}-1\right)+\frac{8 A_{2} y^{3}}{b^{4}}+\frac{8 A_{2} \mu x^{2} y}{a^{4}}\right], \\
\tau_{x y} & =-\frac{E z}{1+\mu} \frac{\partial^{2} \omega}{\partial x \partial y}=-\frac{E z}{1+\mu}\left[\frac{4 A_{2} x}{a^{2}}\left(\frac{x^{2}}{a^{2}}+\frac{y^{2}}{b^{2}}-1\right)+\frac{8 A_{2} x y^{2}}{a^{2} b^{2}}\right] .
\end{aligned}
$$

According to formulas (18) and (19), the internal stress and bending moment of the elliptical rock stratum reach the maximum at point $C$. Therefore, the crack appears first at point $\mathrm{C}$. Then the crack propagates along the periphery.

3.3. Analysis of Failure and Instability Criteria of Rock Stratum. The failure of rock stratum is related to the value of principal stress of any point, and the relationship between the principal stress of any point and the stress component of the point is as follows:

$$
\sigma_{1,3}=\frac{\sigma_{x}+\sigma_{y}}{2} \pm \sqrt{\left(\frac{\sigma_{x}-\sigma_{y}}{2}\right)^{2}+\tau_{x y}^{2}}
$$

where $\sigma_{1}$ is the maximum principal stress and $\sigma_{3}$ is the minimum principal stress.

According to the characteristics that the rock stratum can bear the least tensile stress, the principal stress is compared with the tensile strength of the roof rock mass. If the tensile stress exceeds the tensile strength of the rock mass, the roof will break. According to equation (20), the criterion of rock fracture is as follows:

$$
\max \left\{\sigma_{1}, \sigma_{3}\right\}>\sigma_{T},
$$

where $\sigma_{T}$ is the ultimate tensile strength of rock mass.

Bringing equations (10) and (20) into equation (21), the rock failure criteria can be obtained as follows:

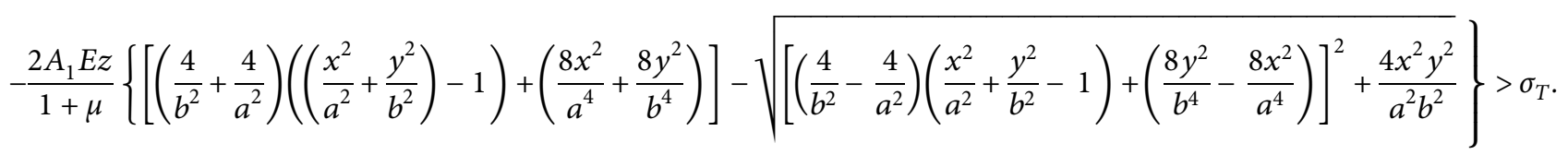

Equation (22) is the failure criterion of the rock stratum. According to equation (22), the fracture of the rock stratum is related to the advancing distance of the working face, the length of the working face, the fracture angle, the elastic modulus, the thickness of the rock stratum, and the overlying load.

The upper surface of rock stratum is compressed and the lower surface is tensioned. According to the characteristics of rock bearing small tensile force, the failure occurs at the lower surface of rock. Therefore, failure is most likely to occur in the center of rock stratum. According to the criterion formula (22), when the tensile stress of the rock just exceeds the ultimate tensile strength of the rock, the advancing distance of the working face can be obtained when the roof rock is damaged. Through derivation and calculation, the advancing distance $L$ of the working face when the stress shell just loses stability is obtained as follows:

$$
L=2 \sqrt{\frac{-c_{2}+\sqrt{c_{2}^{2}+12\left[\sigma_{T}\right] c_{1}}}{2 c_{1}}}+h_{0} \cot \theta_{1}
$$

where $c_{1}$ and $c_{2}$ are constants, respectively. The expression is as follows:

$$
\begin{aligned}
& c_{1}=\frac{3 \gamma \mathrm{H} \mu}{h^{2}\left(\left(L_{0} / 2-h_{0} / 2\right)\left(\cot \theta_{2}+\cot \theta_{3}\right)^{2}\right.}-\frac{3\left[\sigma_{T}\right]}{\left(\left(L_{0} / 2-h_{0} / 2\right)\left(\cot \theta_{2}+\cot \theta_{3}\right)^{4}\right.}, \\
& c_{2}=\frac{3 \gamma \mathrm{H}}{h^{2}}-\frac{22\left[\sigma_{T}\right]}{\left(\left(L_{0} / 2-h_{0} / 2\right)\left(\cot \theta_{2}+\cot \theta_{3}\right)^{2}\right.} .
\end{aligned}
$$




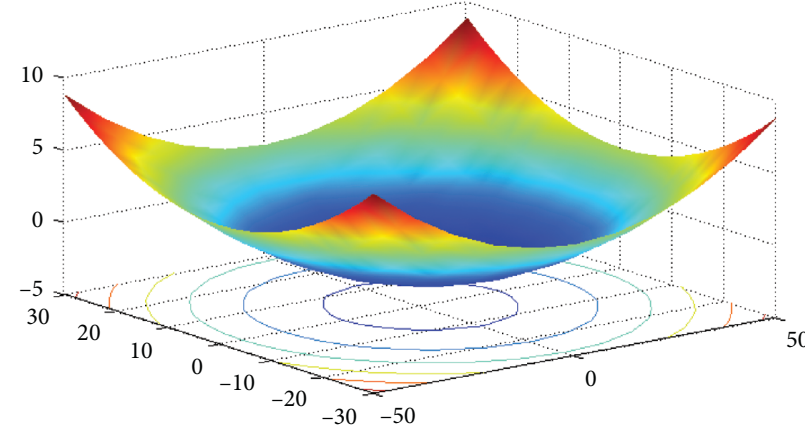

(a)

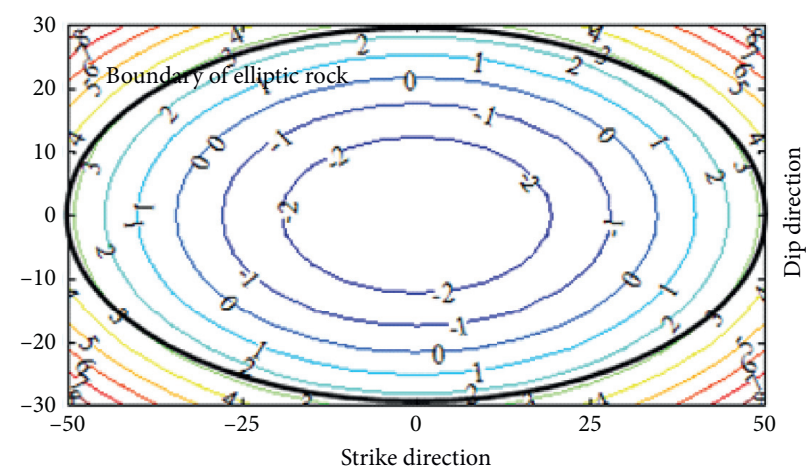

(b)

Figure 6: The stress value of the lower surface of the fixed support. (a) Stereogram of stress distribution. (b) Stress contour.

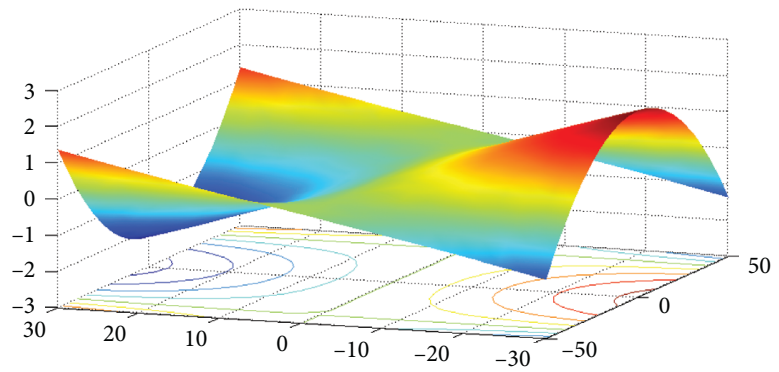

(a)

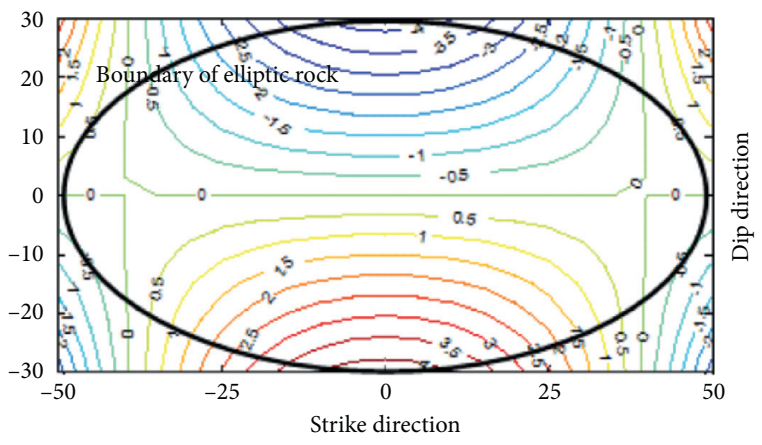

(b)

FIgURE 7: The stress value of the lower surface of the simply supported elliptic plate structure. (a) Stereogram of stress distribution. (b) Stress contour.

\section{Engineering Practice Analysis}

According to 12328 working face of a coal mine, the buried depth of coal seam is $650.4 \mathrm{~m}$, and there is sandstone with thickness of about $15.79 \mathrm{~m}$ at $67.13 \mathrm{~m}$ away from coal seam. According to the actual rock mechanics parameters and roof conditions, the following parameters can be obtained: $a=100 \mathrm{~m}, b=60 \mathrm{~m}, E=4.5 \times 10^{4} \mathrm{MPa}, h=16 \mathrm{~m}, \mu=0.3$, $q=12.4 \mathrm{MPa}, \theta_{1}=60^{\circ}$, and $\theta_{2}=65^{\circ}$. Under the gravity action of overlying rock mass, the upper surface of rock stratum is compressed and the lower surface is tension. Substituting the data of mechanical parameters into equation (20), the contour of maximum principal stress on the lower surface of rock stratum is drawn by using software, as shown in Figures 6 and 7.

Figure 6 shows the maximum principal stress on the lower surface of the thin rock plate with four edges fixed. Under the action of overlying rock mass and gravity, the lower surface of elliptical structure is mainly subjected to tensile stress, and the upper surface is mainly subjected to compressive stress. It can be seen from Figure 6 that the tensile stress in the center of the thin plate is the largest, and the tensile stress in the center is concentrated, so the rock stratum first shows failure in the center of the lower surface.

Figure 7 shows the contour of the maximum principal stress on the lower surface of the simply supported elliptic plate structure. Under this condition, the upper surface of the rock is subjected to compressive stress and the lower surface is subjected to tensile stress. It can be seen from Figure 7 that the maximum tensile stress occurs at the end point of the semiminor axis, and it is prone to failure when the tensile stress exceeds the tensile strength of rock mass.

By analyzing the stress distribution of the lower surface of the elliptical plate under the two mechanical models, it is found that the failure is in the center of the thin rock plate with the largest tensile stress and then at both ends of the semiminor axis. Therefore, the mechanical mechanism and fracture morphology of roof fracture can be found out. The evolution process of roof fracture is shown in Figure 8.

The process of the fracture of the elliptical roof has experienced the following stages: with the advance of the working face, under the action of rock stratum gravity, the elliptical thin plate structure with fixed support around the stope is formed. The roof of the stope starts to break at the middle of the semimajor axis, and the crack extends along the $x$-axis direction to the two ends until it extends to the edge, and finally the bifurcation occurs near the edge. The similar " $X$ " type failure is formed. After the middle position failure, one side simple support and arc length fixed support were formed in the elliptical roof. Under the gravity of the overlying rock mass, the elliptic plate began to break at the end of the semiminor axis, and the crack expanded along the 


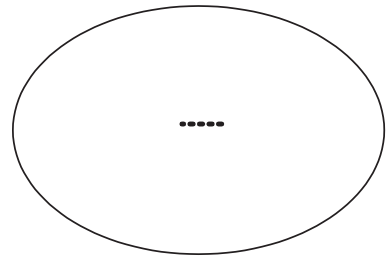

Initial breaking position

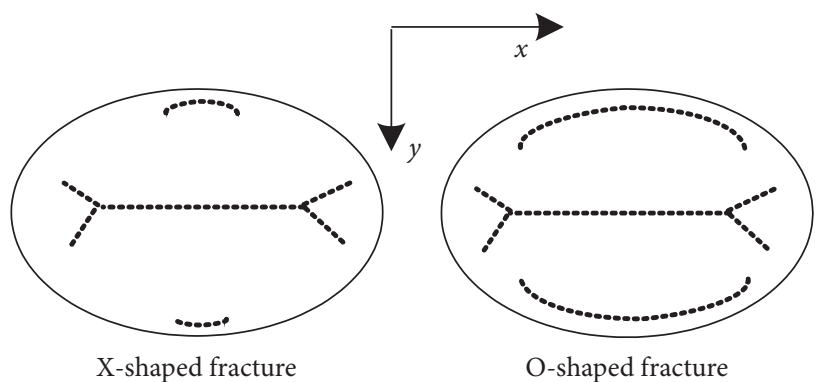

Figure 8: Elliptical structure "X-O" type destroys.

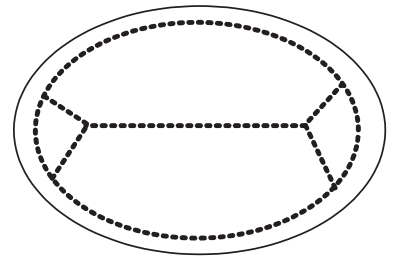

$\mathrm{X}$-O-shaped fracture

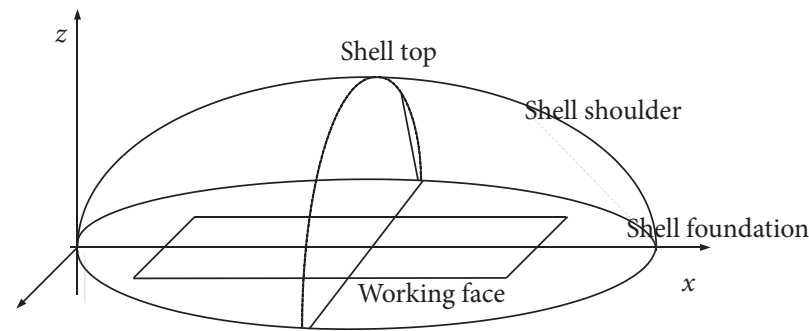

Figure 9: Failure location of stress shell.

arc to both ends, forming two " $\mathrm{C}$ " type failures. The " $\mathrm{C}$ " cracks on both sides were intersected and connected to form "O" type failure, and finally "X-O" failure was formed.

\section{Analysis of the Instability Mode of Stress Shell}

The instability of stress shell is due to the fact that the stress of a part of the stress shell exceeds the ultimate bearing capacity of surrounding rock, which leads to the instability of the whole stress shell of surrounding rock. The mechanical structure characteristics and dynamic evolution process of the stress shell reflect that the top and foundation of the shell are the key positions of the dynamic instability of the stress shell. Therefore, it is easy for the stress shell to lose its stability at these key positions in the evolution process. When the stress at the key position of the stress shell is less than the ultimate strength of the surrounding rock, the stress shell is in a relatively stable state. When the stress of surrounding rock is greater than the ultimate strength of surrounding rock, the surrounding rock at the key position of stress shell will be destroyed, mainly including compression failure, tensile failure, shear failure, and composite failure. As shown in Figure 9, compression failure and composite failure are easy to occur in the shell foundation, shear failure is easy to occur in the shell shoulder, and tensile failure is easy to occur in the shell top. Usually, the tensile strength of rock is less than the shear strength, and the shear strength is less than the compressive strength, so it can be seen that the tensile part of the surrounding rock is most prone to failure. The mechanical analysis of the stress shell in this paper shows that the stress shell is most likely to be tensile failure at the top of the shell. After the failure of the roof rock, the stress shell is unstable, which leads to the redistribution of the surrounding rock stress and the stress transfer to the deep part of the coal and rock mass. When the stress shell is unstable at the top of the shell, it will cause a large area of roof pressure, rock burst, and other severe mine pressure behaviors; at the same time, coal and rock dynamic disasters may occur. The peak value of abutment pressure and the width of shell foundation will reach the maximum value, which will easily lead to further failure of shell foundation. Comprehensive analysis shows that the main instability mode of stress shell is "tensile failure at the top of shell."

\section{Conclusion}

Based on the thin plate theory, the mechanical model of the elliptical structure of the basic roof is constructed. Under the action of the stress shell, the elliptical rock structure is first a fixed-supported boundary, and then a simply supported boundary appears with the fracture of the rock. Through theoretical analysis, the failure criterion of stress shell is obtained. The fracture of the rock stratum is related to the advancing distance of the working face, the length of the working face, the fracture angle, the elastic modulus, the thickness of the rock stratum, and the overlying load.

The "X-O" failure mode is obtained. First, cracks begin to appear at the middle point of the semimajor axis of the elliptical rock stratum, and the cracks propagate along the two ends of the semimajor axis and bifurcate to form " $\mathrm{X}$ " type fracture near the boundary. Then the fracture begins at the end of the semiminor axis, and the crack extends along the arc direction, forming two symmetrical " $C$ " fracture modes. The cracks on both sides connect to form "O" fracture mode and finally form "X-O" fracture mode.

Compression failure and composite failure are easy to occur in the shell foundation, shear failure is easy to occur in the shell shoulder, and tensile failure is easy to occur in the shell top. The mechanical analysis of the stress shell shows 
that the stress shell is most likely to be tensile failure at the top of the shell. After the failure of the roof rock, the stress shell is unstable, which leads to the redistribution of the surrounding rock stress and the stress transfer to the deep of the coal and rock mass. It is revealed that the main instability form of the stress shell is the tensile failure of the bedrock at the top of the shell, which finally leads to the instability of the whole stress shell.

\section{Data Availability}

The data used to support the findings of this study are available from the corresponding author upon request.

\section{Conflicts of Interest}

The authors declare that they have no conflicts of interest.

\section{Acknowledgments}

The research described in this paper was financially supported by State Key Laboratory of Mining Response and Disaster Prevention and Control in Deep Coal Mine Research Fund (SKLMRDPC20KF09).

\section{References}

[1] Y. Zhang, J. Cheng, X. Wang, Z.-J. Feng, and J. Ming, "Thin plate model analysis on roof break of up-dip or down-dip mining stope," Journal of Mining \& Safety Engineering, vol. 27, no. 4, pp. 487-493, 2010.

[2] X. Wang and F.-b. Meng, "Statistical analysis of large accidents in China's coal mines in 2016," Natural Hazards, vol. 92, no. 1, pp. 311-325, 2018.

[3] H. Shi and F. Jiang, "Structural theories of overlying strata in longwall faces and their new development," Journal of Shandong University of Science and Technology, vol. 24, no. 1, pp. 21-25, 2005.

[4] Q. Ma, X. Zhao, and Z. Song, "Break of main roof ahead of workface and ground pressure," Journal Of China Coal Society, vol. 26, no. 5, pp. 473-477, 2001.

[5] X. Liang, G. Liu, and J. Zhao, "Definition and analysis of arching action in underground rock engineering," Journal of Hohai University:Natural Sciences, vol. 33, no. 3, pp. 314-317, 2005.

[6] L. Shi, D. Xu, Y. Wang, M. Qiu, and J. Hao, "A novel conceptual model of fracture evolution patterns in the overlying strata during horizontal coal seam mining," Arabian Journal of Geosciences, vol. 12, no. 10, pp. 1-9, 2019.

[7] J. Chen, L. Zhou, and B. Xia, "Numerical investigation of 3D distribution of mining-induced fractures in response to longwall mining," Natural Resources Research, vol. 30, no. 1, pp. 889-916, 2021.

[8] R. Kumar, A. K. Singh, and A. K. Mishra, "Underground mining of thick coal seams," International Journal of Mining Science and Technology, vol. 25, no. 6, pp. 885-896, 2015.

[9] S. R. Wang, X. G. Wu, Y. H. Zhao, P. Hagan, and C. Cao, "Evolution characteristics of composite pressure-arch in thin bedrock of overlying strata during shallow coal mining," International Journal of Applied Mechanics, vol. 11, no. 3, pp. 195-208, 2019.

[10] S. Zhu, R. Liu, S. Zhang, and D. Hu, "Characteristics of deformation and failure of deep coal seam floor affected by fully mechanized mining," Geotechnical Testing Journal, vol. 39 , no. 1, pp. 1-12, 2016.

[11] X. Jia and Y. Zhai, "The review of ground pressure theory of thin slab in coal mining and its application," Ground Pressure and Strata Control, vol. 3, no. 4, pp. 22-25, 1999.

[12] J. Wang, J. Zhang, X. Gao, J. Wen, and Y. Gu, "Fracture mode and evolution of main roof stratum above longwall fully mechanized top coal caving in steeply inclined thick coal seam (I) - initial fracture," Journal of China Coal Society, vol. 40, no. 6, pp. 1353-1360, 2015.

[13] H. Lin, S. Li, L. Cheng, and W. Zhang, "Key layer distinguishing method of overlying strata based on the thin slab theory," Journal of China Coal Society, vol. 33, no. 10, pp. 1081-1085, 2008.

[14] X. Li, F. Gao, and W. Zhong, "Analysis of fracturing mechanism of stope roof based on plate model," Journal of Mining \& Safety Engineering, vol. 25, no. 2, pp. 180-183, 2008.

[15] X. Zhang, Y. Jiang, and S. Sugimoto, "Anti-plane dynamic response of a non-circular tunnel with imperfect interface in anisotropic rock mass," Tunnelling and Underground Space Technology, vol. 87, pp. 134-144, 2019.

[16] W. Liu, J. Xu, and Z. Wang, "Experimental research on damage characteristics and safety damage threshold of jointed caverns based on acoustic emissions," Geomechanics and Geophysics for Geo-Energy and Geo-Resources, vol. 7, no. 3, pp. 1-14, 2021.

[17] G. X. Xie, J. C. Chang, and K. Yang, "Investigations into stress shell characteristics of surrounding rock in fully mechanized top-coal caving face," International Journal of Rock Mechanics and Mining Sciences, vol. 46, no. 1, pp. 172-181, 2009.

[18] G. Xie, "Influence of mining thickness on mechanical characteristics of working face and surrounding rock stress shell," Journal of China Coal Society, vol. 31, no. 1, pp. 6-11, 2006.

[19] G. Xie and L. Wang, "Lithologic effect on the mechanical characteristics of mining-induced stress shell," Journal of China Coal Society, vol. 38, no. 1, pp. 44-49, 2013.

[20] J. Wu, Elasticity, Higher Education Press, Beijing, China, 2001. 